\title{
MODEL SELECTION METHOD FOR EFFICIENT SIGNALS PROCESSING FROM DISCRETE DATA
}

\author{
Evgeny A. Pchelintsev ${ }^{(a)}$, Serguei M. Pergamenshchikov ${ }^{(b)}$ \\ (a),(b) International Laboratory of Statistics of Stochastic Processes and Quantitative Finance, Tomsk State University, \\ 36 Lenin Avenue, Tomsk, Russia \\ (b) Laboratory of Mathematics Raphael Salem, University of Rouen Normandy, \\ 12 Avenue de l'Universite, Saint Etienne du Rouvray, France \\ ${ }^{(a)}$ evgen-pch@yandex.ru, ${ }^{(b)}$ serge.pergamenchtchikov@,univ-rouen.fr
}

\begin{abstract}
The paper considers the problem of robust adaptive efficient estimating of a periodic signal modeled by a continuous time regression model with the dependent noises given by a non-Gaussian Ornstein-Uhlenbeck process with Levy subordinator in the case when continuous observation cannot be provided and only discrete time measurements are available. Adaptive model selection procedure, based on the improved weighted least square estimates, is proposed. Under some conditions on the noise distribution, sharp oracle inequality for the robust risk has been proved and the robust efficiency of the model selection procedure has been established. The numerical analysis results are given.
\end{abstract}

Keywords: periodic signals, model selection, improved weighted least squares estimates, non-parametric regression, Ornstein-Uhlenbeck process, robust quadratic risk, sharp oracle inequality, asymptotic efficiency

\section{INTRODUCTION}

In problems of signal processing in the informationcommunication complexes they successfully use the continuous time models specified by stochastic differential equations

$$
d y_{t}=S(t) d t+d \xi_{t}, \quad 0 \leq t \leq n,
$$

where $S(t)$ is an unknown 1-periodic signal, $n$ is the duration of observation and $\left(\xi_{t}\right)_{0 \leq t \leq n}$ is unobserved colour noise. The development of the identification procedures is most often based on the assumption that the noises in the equations are specified by the Brownian motion processes, i.e. we obtain the wellknown "signal+white noise" model which is very popular in statistical radio-physics (see, for example, Ibragimov and Khasminskii 1981; Kassam 1988; Chernoyarov et al. 2015 and etc.). If the noise disturbances acting on the dynamic system are nonGaussian and include, for example, some pulse component, then the efficiency of identification algorithms may decrease. Therefore, on the condition that the noise disturbances have more complicated nature, the properties of the decision procedures need an additional investigation. This paper considers the problem of estimating the signal $S$ in the continuous time regression from the Equation (1) with the noises of pulse type, specified by a non-Gaussian OrnsteinUhlenbeck process which obeys the equations

$d \xi_{t}=a \xi_{t} d t+d u_{t}$,
$u_{t}=\tilde{\mathrm{n}}_{1} w_{t}+\tilde{\mathrm{n}}_{2} z_{t}$ and $z_{t}=x *(\mu-\tilde{\mu})_{t}$,

where $a$, $\tilde{\mathrm{n}}_{1}$ and $\tilde{\mathrm{n}}_{2}$ are some unknown constants, $\left(w_{t}\right)_{t \geq 0}$ is a standard Brownian motion, $\mu(d s d x)$ is a jump measure with deterministic compensator $\tilde{\mu}(d s d x)=d s \Pi(d x), \Pi(\cdot)$ is a Levy measure, i.e. some positive measure on $R_{*}=R \backslash\{0\}$ such that $\Pi\left(x^{2}\right)=1$ and $\Pi\left(x^{6}\right)<\infty$. Here we use the notation $\Pi\left(|x|^{m}\right)=\int_{R_{*}}|y|^{m} \Pi(d y)$. Note that the Levy measure $\Pi\left(R_{*}\right)$ could be equal to $+\infty$. We use the notation $*$ for the stochastic integrals with respect to random measures (see Cont and Tankov 2004, Chs. 2 and 3), i.e.

$x *(\mu-\tilde{\mu})_{t}=\int_{0}^{t} \int_{R_{*}} y(\mu-\tilde{\mu})(d s, d y)$.

It should be noted that if $a=0$, then we obtain the Levy regression model considered in (Pchelintsev, Pchelintsev, and Pergamenshchikov 2018). In the case when $\Pi(\cdot)=0$ we obtain the well-known Gaussian Ornstein-Uhlenbeck regression model introduced in (Hopfner and Kutoyants 2009, 2010). The model in the Equations (1) - (2) in which the jump process $\left(z_{t}\right)_{t \geq 0}$ defined by the compound Poisson process was studied in (Konev and Pergamenshchikov 2012, 2015). However, the compound Poisson processes can describe only the large noise impulses of small fixed frequency, 
but the telecommunication and location systems may have the impulse noises with any frequency without any condition. We note also that in the papers (Konev and Pergamenshchikov 2012, 2015) the proposed statistical procedures are based on the classical weighted least square estimators.

The problem is to estimate the unknown signal $S$ in the model (1) on the basis of incomplete observations $\left(y_{t_{j}}\right)_{0 \leq j \leq n p}, t_{j}=j / p$ and $p>2$ is an odd number depending on $n$.

The goal of this paper is to develop a new improved adaptive robust efficient signal estimation methods for the non-Gaussian Ornstein-Uhlenbeck noise based on the general Levy processes with unknown distribution $Q$. We assume that this distribution belongs to the class $Q_{n}{ }^{*}$ defined as a family of all these distributions for which the parameters satisfy the inequalities $-a_{*} \leq a<0, \tilde{\mathrm{n}}_{1} \geq \varsigma_{*}$ and $\tilde{\mathrm{n}}_{1}^{2}+\tilde{\mathrm{n}}_{2}^{2} \leq \varsigma^{*}$ with some fixed positive bounds. The quality of an estimate $\hat{S}_{n}$ of the unknown signal $S$, i.e. some function of $\left(y_{t_{j}}\right)_{0 \leq j \leq n p}$, will be measured with the robust quadratic risk

$\mathrm{R}^{*}\left(\hat{S}_{n}, S\right)=\sup _{Q \in Q_{n}^{*}} \mathrm{R}_{Q}\left(\hat{S}_{n}, S\right)$

where

$\mathrm{R}_{Q}\left(\hat{S}_{n}, S\right):=\mathbf{E}_{Q, S}\left\|\hat{S}_{n}-S\right\|^{2}$ and $\|S\|^{2}=\int_{0}^{1} S^{2}(t) d t$.

Here $\mathbf{E}_{\mathrm{Q}, \mathrm{S}}$ is the expectation with respect to the distribution $\mathbf{P}_{\mathrm{Q}, \mathrm{S}}$ of the process in the Equation (1) with a fixed distribution $Q$ of the noise $\left(\xi_{t}\right)_{0 \leq t \leq n}$ and a given function $S$.

\section{IMPROVED ESTIMATES}

One of the most customarily used methods in the identification theory is the least squares one (LSE). However we can improve (as compared with LSE) the estimation precision using of Stein approach (Stein 1981). This approach to the estimation in the regression models has been developed in (Fourdrinier and Pergamenshchikov 2010, Konev and Pergamenshchikov 2010). At that the gaussianity or spherical symmetricity of distribution of the process under observation plays a key role. In this paper we show that the Stein approach can be used to improve the quality of signal processing observed in the transmission channel with the dependent pulse type noises defined by non-Gaussian Ornstein-Uhlenbeck processes with unknown correlation properties. For this we need to use the modifications of the James - Stein estimators proposed in (Pchelintsev 2013, Konev, Pergamenshchikov, and Pchelintsev 2014) for parametric estimation problems.
For estimating the unknown signal $S$ in the Equation (1) we consider it's Fourier expansion. Let $\left(\phi_{j}\right)_{j \geq 1}$ be a trigonometric basis in $\mathbf{L}_{2}[0,1]$. We extend these functions by the periodic way on $R$, i.e. $\phi_{j}(t)=\phi_{j}(t+1)$ for any $t \in R$. We will use such basis that the restrictions of the functions $\left\{\phi_{j}\right\}_{1 \leq j \leq p}$ on the sampling lattice $T_{p}=\left\{t_{1}, \ldots, t_{p}\right\}, \quad t_{j}=j / p$, form an orthonormal basis in the Hilbert space $R^{T_{p}}$ with the inner product

$(x, y)_{p}=\frac{1}{p} \sum_{j=1}^{p} x\left(t_{j}\right) y\left(t_{j}\right)$ for $x, y \in R^{T_{p}}$,

i.e. $\left(\phi_{i}, \phi_{j}\right)_{p}=1_{\{i=j\}}$ for any odd $p \geq 3,1_{A}$ is the indicator of the set $A$. We write the discrete Fourier expansion of the unknown signal $S$ on the lattice $T_{p}$ in the form

$S(t)=\sum_{j=1}^{p} \theta_{j, p} \phi_{j}(t)$ and $\theta_{j, p}=\left(S, \phi_{j}\right)_{p}$.

The first step in estimation procedure consists in estimating the Fourier coefficients $\theta_{j, p}$ from discrete data by the formulae

$\hat{\theta}_{j, p}=\frac{1}{n} \int_{0}^{n} \psi_{j, p}(t) d y_{t}$ with $\psi_{j, p}=\sum_{k=1}^{n p} \phi_{j}\left(t_{k}\right) 1_{\left\{t_{k-1}<t \leq t_{k}\right\}}$

Further, for the first $d \leq p$ Fourier coefficients in Equations (7) we use the improved estimation method proposed for parametric models in (Pchelintsev 2013, Konev, Pergamenshchikov, and Pchelintsev 2014). To this end we set $\tilde{\theta}_{p}=\left(\hat{\theta}_{j, p}\right)_{1 \leq j \leq d}$. In the sequel, we will use the norm $|x|_{d}^{2}=\sum_{j=1}^{d} x_{j}^{2}$ for any vector $x=\left(x_{j}\right)_{1 \leq j \leq d}$ from $R^{d}$. We define the shrinkage estimators as

$\theta_{j, p}^{*}=(1-g(j)) \hat{\theta}_{j, p}$,

where $\quad g(j)=\frac{c_{n}}{\left|\tilde{\theta}_{p}\right|_{d}} 1_{\{1 \leq j \leq d\}}, \quad c_{n} \quad$ is some known parameter such that $c_{n} \approx d / n$ as $n \rightarrow \infty$ (Pchelintsev and Pergamenshchikov 2019). Now we introduce a class of shrinkage weighted least squares estimates for $S$ as

$S_{\gamma}^{*}(t)=\sum_{j=1}^{p} \gamma(j) \theta_{j, p}^{*} \psi_{j, p}(t)$, 
where the weights $\gamma \in R^{n}$ belong to some finite set $\Gamma$ from $[0,1]^{p}$ defined in (Konev and Pergamenshchikov 2015, Pchelintsev and Pergamenshchikov 2018).

To compare the non-asymptotic accuracy of the proposed shrinkage estimate in Equations (10) and LSE $\hat{S}_{\gamma}(t)$ from (Konev and Pergamenshchikov 2015, Equation (3.10)) we denote the difference of their quadratic risks as $\Delta_{Q}(S):=\mathrm{R}_{Q}\left(S_{\gamma}^{*}, S\right)-\mathrm{R}_{Q}\left(\hat{S}_{\gamma}, S\right)$. Now for this deviation, we obtain the following result.

Theorem 1. For any $p>\sqrt{d} \log n / c_{n}$ and $r>0$

$\sup \sup \Delta_{Q}(S)<0$

The inequality in Equation (11) means that nonasymptotically, i.e. for any $n \geq n_{0}$ the estimate in the Equation (10) outperforms in mean square accuracy the LSE.

\section{MODEL SELECTION METHOD AND ORACLE INEQUALITY}

The model selection procedure for estimating an unknown signal $S$ in the Equation (1) will be constructed on the basis of a family of improved estimates $\left(S_{\gamma}^{*}\right)_{\gamma \in \Gamma}$. The performance of any estimate $S_{\gamma}^{*}$ will be measured by the empirical squared error

$$
\operatorname{Err}_{p}(\gamma)=\left\|S_{\gamma}^{*}-S\right\|^{2}
$$

In order to obtain a good estimate, we have to write a rule to choose a weight vector $\gamma \in \Gamma$ in the Equation (10). It is obvious, that the best way is to minimize the empirical squared error with respect to $\gamma$. Making use the estimate definition in the Equation (10) and the Fourier transformation of $S$ implies

$$
\operatorname{Err}_{p}(\gamma)=\sum_{j=1}^{p} \gamma^{2}(j)\left(\theta_{j, p}^{*}\right)^{2}-2 \sum_{j=1}^{p} \gamma(j) \theta_{j, p}^{*} \bar{\theta}_{j, p}+\|S\|^{2},
$$

where $\left(\bar{\theta}_{j, p}\right)_{j \geq 1}$ are the Fourier coefficients for the signal $S$ with respect to the orthonormal system of the functions $\left\{\psi_{j, p}\right\}_{1 \leq j \leq p}$. Since these coefficients are unknown, the weight coefficients $\left(\gamma_{j}\right)_{j \geq 1}$ cannot be found by minimizing $\operatorname{Err}_{p}(\gamma)$. To circumvent this difficulty one needs to replace the terms $\theta_{j, p}^{*} \bar{\theta}_{j, p}$ by their estimators of the form

$$
\tilde{\vartheta}_{j, p}=\theta_{j, p}^{*} \hat{\theta}_{j, p}-\frac{\hat{\sigma}_{n}}{n},
$$

where $\hat{\sigma}_{n}$ is the estimate for the noise variance $\sigma_{Q}=\mathbf{E}_{Q} \xi_{j, n}^{2}$ which we choose in the following form

$$
\hat{\sigma}_{n}=\sum_{j=[\sqrt{n}]+1}^{n} \hat{t}_{j, n}^{2} \text { and } \hat{t}_{j, n}=\frac{1}{n} \int_{0}^{n} \phi_{j}(t) d y_{t} .
$$

For this change in the empirical squared error, one has to pay some penalty. Thus, one comes to the cost function of the form

$$
J_{p}(\gamma)=\sum_{j=1}^{p} \gamma^{2}(j)\left(\theta_{j, p}^{*}\right)^{2}-2 \sum_{j=1}^{p} \gamma(j) \tilde{\vartheta}_{j, p}+\delta \hat{P}(\gamma),
$$

where $\delta$ is some positive constant, $\hat{P}(\gamma)$ is the penalty term defined as

$\hat{P}(\gamma)=\frac{\hat{\sigma}_{n}|\gamma|_{p}^{2}}{n}$.

Substituting the weight coefficients, minimizing the cost function

$\gamma^{*}=\operatorname{agrmin}_{\gamma \in \Gamma} J_{p}(\gamma)$

in the Equation (10) leads to the improved model selection procedure

$$
S^{*}=S_{\gamma^{*}}^{*}
$$

It will be noted that $\gamma^{*}$ exists because $\Gamma$ is a finite set. If the minimizing sequence in the Equation (18) $\gamma^{*}$ is not unique, one can take any minimizer.

In the case, when the value of $\sigma_{Q}$ is known, one can take $\hat{\sigma}_{n}=\sigma_{Q}$ and $P(\gamma)=\sigma_{Q}|\gamma|_{p}^{2} n^{-1}$.

Theorem 2. For any $n \geq 2$ and $0<\delta<1 / 2$, the robust risks defined in the Equation (4) of estimate in the Equation (19) for continuously differentiable function $\mathrm{S}$ satisfies the oracle inequality

$\mathrm{R}^{*}\left(S^{*}, S\right) \leq \frac{1+5 \delta}{1-\delta} \min _{\gamma \in \Gamma} \mathrm{R}^{*}\left(S_{\gamma}^{*}, S\right)+\frac{B_{n}^{*}}{n \delta}$,

where the term $B_{n}^{*}$ is independent of $\mathrm{S}$ and such that $B_{n}^{*} n^{-\varepsilon} \rightarrow 0$ as $n \rightarrow \infty$ for any $\varepsilon>0$.

The inequality (20) allows us to establish that the procedure in the Equation (19) is optimal in the oracle inequalities sense. This property enables to provide asymptotic efficiency in the adaptive setting, i.e. when information about the signal regularity is unknown. 


\section{MONTE CARLO SIMULATIONS}

In this section we report the results of a Monte Carlo experiment to assess the performance of the proposed model selection procedure in the Equation (19). In the Equation (1) we choose 1-periodic signal $S$ which is defined as $S(t)=t \sin (2 \pi t)+t^{2}(1-t) \cos (2 \pi t)$, for $0 \leq t \leq 1$. We simulate the Equation (1) with the Ornstein-Uhlenbeck noise process defined as

$d \xi_{t}=-\xi_{t} d t+0.5 d w_{t}+0.5 d z_{t}$

where $z_{t}=\sum_{j=1}^{N_{t}} Y_{j} \quad N_{t}$ is a Poisson process with the intensity $\lambda=1$ and $\left(Y_{j}\right)_{j \geq 1}$ is i.i.d. Gaussian $(0,1)$. We use the model selection procedure defined in the Equation (19) with the weights proposed in (Konev and Pergamenshchikov 2015): $k^{*}=100+\sqrt{\ln n}, \varepsilon=1 / \ln n$ and $m=\left[1 / \varepsilon^{2}\right]$. We used the cost function with $\delta=(3+\ln n)^{-2}$. We define the empirical risk as $\bar{R}(\tilde{S}, S)=\frac{1}{p} \sum_{j=1}^{p} \hat{\mathbf{E}}\left(\tilde{S}_{n}\left(t_{j}\right)-S\left(t_{j}\right)\right)^{2}$ and $\hat{\mathbf{E}}\left(\tilde{S}_{n}(\cdot)-S(\cdot)\right)^{2}$ $=\frac{1}{N} \sum_{l=1}^{N}\left(\tilde{S}_{n}^{l}(\cdot)-S(\cdot)\right)^{2} \quad$ with the frequency of observations $p=100001$ and numbers of replications $N=1000$.

Table 1 gives the values for the sample risks of the improved estimate Equation (19) and the model selection procedure based on the weighted LSE from (Konev and Pergamenshchikov 2015) for different numbers of observation period $n$. Table 2 gives the values for the sample risks of the model selection procedure based on the weighted LSE from (Konev and Pergamenshchikov 2015) and it's improved version for different numbers of observation period $n$.

Table 1: The sample quadratic risks for different optimal $\gamma$

\begin{tabular}{|c|c|c|c|c|}
\hline$n$ & 100 & 200 & 500 & 1000 \\
\hline $\bar{R}\left(S_{\gamma^{*}}^{*}, S\right)$ & 0.0289 & 0.0089 & 0.0021 & 0.0011 \\
\hline $\bar{R}\left(\hat{S}_{\hat{\gamma}}, S\right)$ & 0.0457 & 0.0216 & 0.0133 & 0.0098 \\
\hline$\frac{\bar{R}\left(\hat{S}_{\hat{\gamma}}, S\right)}{\bar{R}\left(S_{\gamma^{*}}^{*}, S\right)}$ & 1.6 & 2.4 & 6.3 & 8.9 \\
\hline
\end{tabular}

From the Table 2 for the same weights $\gamma$ with various observations numbers $n$ we can conclude that theoretical result on the improvement effect is confirmed by the numerical simulations. Moreover, from Table 1 and Figure 1, we can see that the proposed method has the higher estimation quality then LSE.

On the figures the bold line is the signal $S$, the continuous line is the model selection procedure based on the least squares estimators $\hat{S}$ and the dashed line is the improved model selection procedure $S^{*}$.
Table 2: The sample quadratic risks for the same optimal $\hat{\gamma}$

\begin{tabular}{|c|c|c|c|c|}
\hline$n$ & 100 & 200 & 500 & 1000 \\
\hline $\bar{R}\left(S_{\gamma^{*}}^{*}, S\right)$ & 0.0391 & 0.0159 & 0.0098 & 0.0066 \\
\hline $\bar{R}\left(\hat{S}_{\hat{\gamma}}, S\right)$ & 0.0457 & 0.0216 & 0.0133 & 0.0098 \\
\hline$\frac{\bar{R}\left(\hat{S}_{\hat{\gamma}}, S\right)}{\bar{R}\left(S_{\gamma^{*}}^{*}, S\right)}$ & 1.2 & 1.4 & 1.3 & 1.5 \\
\hline
\end{tabular}

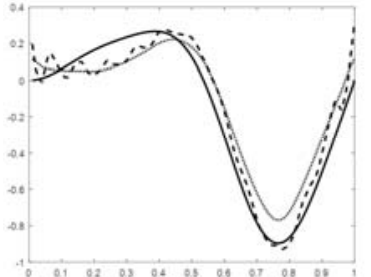

a) $n=500$

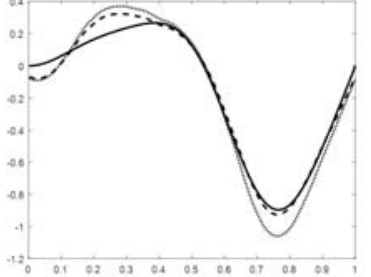

b) $n=1000$
Figure 1: Behavior of the regression function and its estimates

\section{ASYMPTOTIC EFFICIENCY}

We define the following functional Sobolev ball

$$
W_{k, \mathbf{r}}=\left\{f \in C_{p}^{k}[0,1]: \sum_{i=0}^{k}\left\|f^{(i)}\right\|^{2} \leq \mathbf{r}\right\},
$$

where $\mathbf{r}>0$ and $k \geq 1$ are some unknown parameters, $C_{p}^{k}[0,1]$ is the space of $k$ times differentiable 1-periodic functions such that for any $0 \leq i \leq k-1$ : $f^{(i)}(0)=f^{(i)}(1)$. In order to formulate our asymptotic results we set

$v_{n}=\frac{n}{\varsigma^{*}}, l_{k}(\mathbf{r})=((2 k+1) \mathbf{r})^{1 /(2 k+1)}\left(\frac{k}{\pi(k+1)}\right)^{2 k /(2 k+1)}$

and we denote by $\Sigma_{n}$ of all estimates $\hat{S}_{n}$ of $S$ from discrete observations of the process in the Equation (1). Also we denote by $Q^{*}$ the distribution of the process in the Equation (1) with $\xi_{t}=\varsigma^{*} w_{t}$, i.e. white noise model with the intensity $\varsigma^{*}$.

Theorem 3. Assume that $Q^{*} \in Q_{n}^{*}$. Then the robust risk defined in the Equation (4) admits the following asymptotic lower bound

$$
\liminf _{n \rightarrow \infty} \inf _{\hat{S}_{n} \in \Sigma_{n}} v_{n}^{2 k /(2 k+1)} \sup _{S \in W_{k, \mathrm{r}}} \mathrm{R}^{*}\left(\hat{S}_{n}, S\right) \geq l_{k}(\mathbf{r}) .
$$

This lower bound is sharp in the following sense.

Theorem 4. Assume that $Q^{*} \in Q_{n}^{*}$ and there exists $\varepsilon>0$ such that $\lim _{n \rightarrow \infty} n^{5 / 6+\varepsilon} / p=0$. Then the robust risk defined in the Equation (4) for the estimating 
procedure in the Equation (19) has the following asymptotic upper bound

$\limsup _{n \rightarrow \infty} v_{n}^{2 k /(2 k+1)} \sup _{S \in W_{k, \mathbf{r}}} \mathrm{R}^{*}\left(S^{*}, S\right) \leq l_{k}(\mathbf{r})$.

Theorems 3 and 4 imply that the model selection procedure $S^{*}$ is efficient and the parameter $l_{k}(\mathbf{r})$ defined in the Equations (23) is the Pinsker constant in this case (Pchelintsev and Pergamenshchikov 2018, 2019).

\section{CONCLUSION}

In this paper, we considered the problem of nonparametric signal processing on the basis of the discrete time observations with the dependent nonGaussian impulse noises modelled by OrnsteinUhlenbeck processes. We developed adaptive efficient statistical model selection procedure based on the improved methods which outperforms the LSE in mean square accuracy. The obtained theoretical results are confirmed by the numerical simulation. We obtained the adaptive efficiency property for the proposed statistical method, which means that we provide the best mean square accuracy without using the smoothness information about the form of unknown signal. We studied the accuracy properties for the proposed method on the basis of the robust approach, i.e. uniformly over all possible unknown noise distributions. This allows us to synthesize the statistical algorithms possessing the high noise immunity properties. The results (their satisfactory concordance with the corresponding experimental data) can be used for the estimation of the signals. Such problems are of a great importance in the fields of radio-and-hydroacoustic communications and positioning, radio-and-hydrolocation, etc. (see, for details, Chernoyarov, Kutoyants, and Marcokova 2018 and references therein).

\section{ACKNOWLEDGMENTS}

This work was supported by the RSF, research project no. 17-11-01049.

\section{REFERENCES}

Chernoyarov O.V., Vaculik M., Shirikyan A. and Salnikova A.V., 2015. Statistical Analysis of Fast Fluctuating Random Signals with Arbitrary Function Envelope and Unknown Parameters. Communications - Scientific Letters of the University of Zilina, 17 (1a), 35-43.

Chernoyarov O.V., Kutoyants Yu. A. and Marcokova M., 2018. On frequency estimation for partially observed system with small noises in state and observation equations. Communications Scientific Letters of the University of Zilina, 20 (1), 66-71.

Cont R. and Tankov P., 2004. Financial Modelling with Jump Processes. London: Chapman \& Hall.

Fourdrinier D. and Pergamenshchikov S., 2010. Improved selection model method for the regression with dependent noise. Annals of the Institute of Statistical Mathematics, 59 (3), 435464.

Hopfner R. and Kutoyants Yu.A., 2009. On LAN for parametrized continuous periodic signals in a time-inhomogeneous diffusion. Statistical Decisions, 27 (4), 309-326.

Hopfner R. and Kutoyants Yu.A., 2010. Estimating discontinuous periodic signals in a timeinhomogeneous diffusion. Statistical Inference for Stochastic Processes, 13 (3), 193-230.

Ibragimov I.A. and Khasminskii R.Z., 1981. Statistical Estimation: Asymptotic Theory. New York: Springer.

Kassam S.A., 1988. Signal Detection in Non-Gaussian Noise. New York: Springer.

Konev V.V. and Pergamenshchikov S.M., 2010. General model selection estimation of a periodic regression with a Gaussian noise. Annals of the Institute of Statistical Mathematics, 62, 1083 1111.

Konev V.V. and Pergamenshchikov S.M., 2012. Efficient robust nonparametric estimation in a semimartingale regression model. Annales de l'Institut Henri Poincare (B) Probability and Statistics, 48 (4), 1217-1244.

Konev V.V. and Pergamenshchikov S.M., 2015. Robust model selection for a semimartingale continuous time regression from discrete data. Stochastic Processes and their Applications, 125, 294-326.

Konev V., Pergamenshchikov S. and Pchelintsev E., 2014. Estimation of a regression with the pulse type noise from discrete data. Theory of Probability and Its Applications, 58 (3), 442-457.

Pchelintsev E., 2013. Improved estimation in a nonGaussian parametric regression. Statistical Inference for Stochastic Processes, 16 (1), 15-28.

Pchelintsev E. and Pergamenshchikov S., 2018. Oracle inequalities for the stochastic differential equations. Statistical Inference for Stochastic Processes, 21 (2), 469-483.

Pchelintsev E. and Pergamenshchikov S., 2019. Improved model selection method for an adaptive estimation in semimartingale regression models. Tomsk State University Journal of Mathematics and Mechanics, 58, 14-31.

Pchelintsev E., Pchelintsev V. and Pergamenshchikov S., 2018. Non asymptotic sharp oracle inequalities for the improved model selection procedures for the adaptive nonparametric signal estimation problem. Communications - Scientific Letters of the University of Zilina, 20 (1), 72-76.

Stein C.M., 1981. Estimation of the mean of a multivariate normal distribution. Ann. Statist., 9 (6), 1135-1151.

\section{AUTHORS BIOGRAPHY}

Evgeny PCHELINTSEV was born in Almaty, Kazakhstan. He received his M.Sc. in Mathematics 
from Tomsk State University in 2009, Ph.D. in Applied Mathematics and Statistics from Tomsk State University and Rouen University in 2012. Since 2012 he worked as a teacher of mathematics at several mathematical departments at Tomsk State University. His research interests include statistical modelling and identification of stochastic dynamic systems, soft computing, and simulation

Serguei PERGAMENSHCHIKOV was born in Tomsk, Russia. He received his M.Sc. from Tomsk State University in 1980, Ph.D. in Probability Theory and statistics from Tomsk State University in 1986, Doctor of Sciences in Mathematics from Central Economics and Mathematics Institute of RAS in 1994. His research interests include statistical modelling and identification of stochastic dynamic systems, stochastic differential equations, optimization, and control. 